\title{
钯催化取代吲哚与苯丙酮类化合物的直接偶联构筑咔唑衍生物
}

\author{
周全龙 $\dagger, a, b$ 朱昌垒 ${ }^{\dagger}, a$ 吴 戈 ${ }^{a}$ 张远飞 ${ }^{a}$ \\ 张 敏*, $a$ 苏伟平 $*, a, b$ \\ $\left({ }^{a}\right.$ 中国科学院福建物质结构研究所 结构化学国家重点实验室 福州 350002)

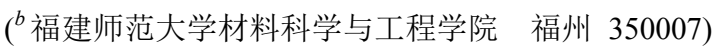

\begin{abstract}
摘要 报道了一种过渡金属钯催化的由取代吲哚与苯丙酮类化合物直接脱氢偶联构筑味唑衍生物的新方法. 反应经历 饱和苯丙酮类化合物的原位脱氢形成烯烃中间体，该中间体直接与吲哚发生反应脱氢环化，省略了底物的预活化，为 咔唑衍生物的合成提供原子和步骤经济的合成路径.
\end{abstract}

关键词＼cjkstart钯催化; 脱氢; 咔唑; $\mathrm{C}-\mathrm{H}$ 键官能团化; 交叉偶联

\section{Construction of Carbazoles by Palladium-Catalyzed Direct Cross- Coupling of Indoles with in situ Generated Aryl Vinyl Ketones}

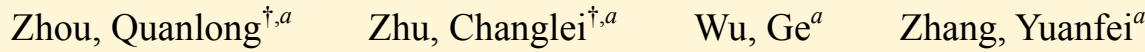 \\ Zhang, Min ${ }^{*, a} \quad \mathrm{Su}$, Weiping ${ }^{*, a, b}$ \\ $\left({ }^{a}\right.$ State Key Laboratory of Structural Chemistry, Fujian Institute of Research on the Structure of Matter, \\ Chinese Academy of Sciences, Fuzhou 350002) \\ $\left({ }^{b}\right.$ College of Materials Science and Engineering, Fujian Normal University, Fuzhou 350007)
}

\begin{abstract}
The synthesis of carbazoles via Pd-catalyzed direct cross-coupling of indoles with in situ generated aryl vinyl ketones by using statured ketones as the olefins source is described. This protocol obviates the need for additional preparation steps of aryl vinyl ketones and therefore opens up a new door to synthesis of carbazoles in an atom- and step-economical fashion.

Keywords palladium-catalyzed; dehydrogenation; carbazoles; $\mathrm{C}-\mathrm{H}$ functionalization; cross-coupling
\end{abstract}

Carbazoles represent an important class of nitrogencontaining heterocyclic compounds, which are ubiquitous as structural motifs in various natural products, pharmaceuticals and biologically active compounds (Figure 1). ${ }^{[1]}$ Traditionally, the most commonly used method for preparation of this kind of molecular framework mainly relies upon nitrene insertion, Fischer indolization, Pummerer cyclization, Dielse-Alder reaction, dehydrogenative cyclization of diarylamines, etc. ${ }^{[2]}$ In view of their significant importance and wide application, synthetic organic chemists are devoted to seek efficient methods for the construction of carbazoles during the past several decades.

The past decades have witnessed the rapid development of transition metal-catalyzed $\mathrm{C}-\mathrm{H}$ bond functionalization reactions, which have evolved into a powerful tool for the synthesis of numerous structurally complex molecules from simple starting materials. ${ }^{[3]}$ Several methods involving $\mathrm{C}-$ $\mathrm{H}$ cleavage and $\mathrm{C}-\mathrm{C} / \mathrm{C}-\mathrm{N}$ formation have been used for synthesis of carbazoles, such as oxidative cyclization of biarylamines ${ }^{[4]}$ and intramolecular $\mathrm{C}-\mathrm{H}$ amination of anilides. ${ }^{[5]}$ Among the various methods developed in recent years, the indole-to-carbazole transformation, which relies upon the construction of a benzene ring into indoles, has attracted widespread attention since it offers a direct and convenient approach to carbazoles from readily available indoles. Substituted carbazoles were obtained via the $[2+$ $2+2]$ cycloaddition of indoles with alkyne. ${ }^{[6]}$ Recently, the synthesis of carbazoles by direct cross-coupling of indoles with alkenes has appeared, ${ }^{[7]}$ and those reactions were proposed to occur via an initially alkenylation of indoles, ${ }^{[8]}$

\footnotetext{
* Corresponding authors. E-mail: wpsu@fjirsm.ac.cn; zhangmin@fjirsm.ac.cn Received May 8, 2017; revised July 6, 2017; published online August 11, 2017. Project supported by the National Natural Science Foundation of China (Nos. 21431008, 21332001, 21602221, u1505242). 国家自然科学基金(Nos. 21431008, 21332001, 21602221, u1505242)资助项目.

共同第一作者(These authors contributed equally to this work).
} 
followed by a Diels-Alder reaction of the 3-alkenyated indoles with the second alkenes.

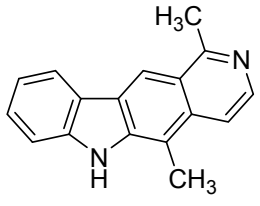

Guatambuinine

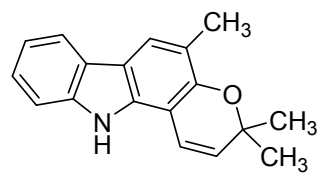

girinimbine

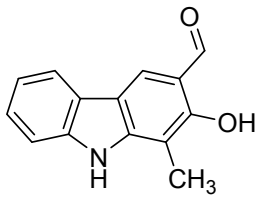

mukonal

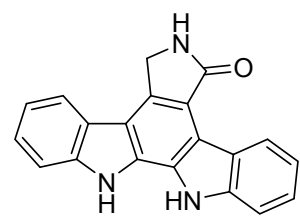

staurosporine aglycone
Figure 1 Biologically active carbazole derivatives

Although there are reports on the direct $\mathrm{C}-\mathrm{H} / \mathrm{C}-\mathrm{H}$ coupling of indoles with alkenes to provide carbazoles, examples using aryl vinyl ketones are much less common. In this context, $\mathrm{Yu}$ and coworkers ${ }^{[9]}$ reported the synthesis of 1,3-disubstituted carbazoles via the reactions of indoles with in-situ generated aryl vinyl ketones using saturated $\beta$-chloroalkyl aryl ketones as the olefins source. Aryl vinyl ketones are not commercially available, typical preparation of aryl vinyl ketones require multistep preparation routes ${ }^{[10]}$ and/or use of stoichiometric reagents such as 2-iodoxybenzoic acid (IBX) ${ }^{[11]}$ and dichloro-5,6-dicyano-1,4-benzoquinone (DDQ). ${ }^{[12]}$ The Pd-catalyzed oxidative dehydrogenation of ketones represents a promising method for the facile synthesis of $\alpha, \beta$-unsaturated ketones, but is still limited to cyclic enones or $\beta$-arylated enones. ${ }^{[13]}$ Consequently, the reactions that combine in situ dehydrogenation to form reactive aryl vinyl ketone intermediates with secondary coupling process are attracting attention from synthetic chemists. Herein, we report an efficient method for the facile syntheses of carbazoles via Pd-catalyzed dehydrogenative cross-coupling of indoles with in situ generated aryl vinyl ketones by using statured ketones as the olefins source.

As our ongoing interest in the development of $\mathrm{C}-\mathrm{H}$ functionalization reactions, ${ }^{[14]}$ we previously have reported several methods for palladium-catalyzed dehydrogenative olefination of (hetero)arenes via olefin intermediates generated in situ from saturated ketones and nitroalkanes. ${ }^{[15]}$ Furthermore, we have achieved the $\beta$-functionalization of saturated ketones by palladium-catalyzed dehydorgenation of ketones in combination with subsequent conjugate addition. ${ }^{[16]}$ These encouraging achievements led us to consider whether the dehydrogenative cross-coupling between indoles and saturated ketones could directly afford carbazoles. The expected transformation which just involves multiple successive $\mathrm{C}-\mathrm{H}$ cleavages and $\mathrm{C}-\mathrm{C}$ formation steps in one pot would provide a fundamentally new approach to carbazoles in an atom- and step-economical fashion.

\section{Results and discussion}

We initially screened a variety of reaction parameters using the reaction of $N$-methyl indole (1a) and propiophenone (2a) as a model system (Table 1). To our delight, the desired $N$-methyl-1,3-dibenzoylcarbazole (3a) was obtained in $77 \%$ yield when the reaction mixture of $1 \mathbf{a}$ and $\mathbf{2 a}$ (10 equiv.) in 1,2-dimethoxyethane (DME) was treated with $\mathrm{Pd}(\mathrm{OAc})_{2}(10 \mathrm{~mol} \%), \mathrm{PCy}_{3}(25 \mathrm{~mol} \%), \mathrm{Ag}_{2} \mathrm{CO}_{3}$ (3.0 equiv.), LiOAc (1.0 equiv.) and TEMPO (2,2,6,6-tetramethylpiperidine- $N$-oxyl) ( 0.4 equiv.) at $110{ }^{\circ} \mathrm{C}$ under 101 $\mathrm{kPa}$ of $\mathrm{O}_{2}$. Control experiments indicated that all the reaction parameters (palladium, copper, ligand, base and additive) were essential to furnish the product in high yield (Entries $2 \sim 6$ ). The use of TEMPO ( 0.4 equiv.) as a co-oxidant led to an increase in yield presumably owing to its ability to accelerate reoxidation of $\mathrm{Pd}(0)$ to $\mathrm{Pd}(\mathrm{II})$ (Entry 4 vs 1). The yield were decreased if the amount of $\mathrm{Ag}_{2} \mathrm{CO}_{3}$ were reduced (Entries $7 \sim 8$ ). Replacing $\mathrm{PCy}_{3}$ with other phosphine ligand such as $\mathrm{PPh}_{3}$, XPhos or Davephos significantly reduced the efficiency of reaction (Entries 9 11). LiOAc also played an irreplaceable role in enhancing the efficiency of the reaction (Entries $12 \sim 15$ ). The yield was decreased significant when this reaction was carried out under air or $\mathrm{N}_{2}$ atmospher, indicating that $\mathrm{O}_{2}$ played a significant role as the oxidant in this reaction (Entries 16 17).

With the optimized conditions in hand, we examined the substrate scope of this reaction with respect to propiophenones. As shown in Table 2, an array of substituted propiophenones underwent smoothly the reaction with $\mathrm{N}$ methylindole (1a) to produce the desired 1,3-disubstituted carbazoles in moderate yields. As a ligand, small adjustments about the amount of $\mathrm{PCy}_{3}$ were required to the reaction conditions in some cases to get better yields (3f, 3k). The variety of substituents tolerated in these cross-couplings, such as alkyl (3a $\sim \mathbf{3 e}, \mathbf{3 g})$, alkoxy (3f, 3h), fluoro (3j), chloro (3k) and trifluoromethyl (3i) provide opportunities for further synthetic elaboration. Unfortunately, other sensitive substituents such as the ester, nitro, and cyano group were ineffective under the established conditions. Notably, heteroaromatic ketones such as 2-propionylthiophene were also appropriate reactants for this transformation (3I).

Next, we evaluated the scope of indoles by using propiophenone (2a) as a coupling partner. As shown in Table 3, the present protocol also proved to be applicable to $N$-Bn and $N-n$-Pr indoles $(\mathbf{4 a}, \mathbf{4 b})$. However, when $N-\mathrm{H}$ indoles were induced to react with $\mathbf{2 a}$ under the established conditions, the desired products were not obtained. The reaction of indoles bearing electron-donating methyl and mothoxyl $(4 c, 4 d)$, or electron-withdrawing fluoro and chloro $(\mathbf{4 e}, \mathbf{4 f})$ substituents on benzene ring underwent the reaction smoothly to provide the desired products. Other electron-withdrawing substituents such as the ester, nitro, and cyano group were ineffective. 
Table 1 Optimization studies of reaction conditions ${ }^{a}$<smiles>Cn1ccc2ccccc21</smiles>

$1 \mathrm{a} 0.2 \mathrm{mmol}$<smiles>CCC(=O)c1ccccc1</smiles>

2a 10 equiv.

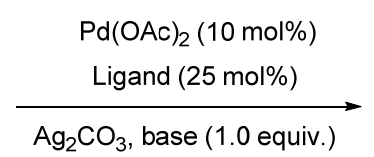

TEMPO, $\mathrm{O}_{2}, \mathrm{DME}, 110^{\circ} \mathrm{C}, 24 \mathrm{~h}$

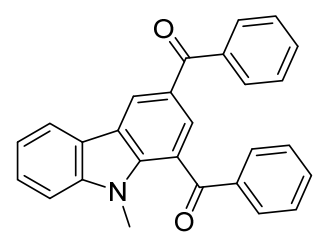

$3 a$

\begin{tabular}{|c|c|c|c|c|c|c|c|c|c|c|c|}
\hline Entry & $\begin{array}{c}\mathrm{Ag}_{2} \mathrm{CO}_{3} / \\
\text { equiv. }\end{array}$ & Ligand & Base & $\begin{array}{l}\text { TEMPO/ } \\
\text { equiv. }\end{array}$ & $\begin{array}{c}\text { Yield }^{b} / \% \\
\text { of } \mathbf{3 a}\end{array}$ & Entry & $\begin{array}{c}\mathrm{Ag}_{2} \mathrm{CO}_{3} / \\
\text { equiv. }\end{array}$ & Ligand & Base & $\begin{array}{l}\text { TEMPO/ } \\
\text { equiv. }\end{array}$ & $\begin{array}{c}\text { Yield }^{b} / \% \\
\text { of } \mathbf{3 a}\end{array}$ \\
\hline 1 & 3.0 & $\mathrm{PCy}_{3}$ & LiOAc & 0.4 & 77 (76) & 10 & 3.0 & Xphos & LiOAc & 0.4 & Trace \\
\hline $2^{c}$ & 3.0 & $\mathrm{PCy}_{3}$ & LiOAc & 0.4 & 0 & 11 & 3.0 & Davephos & LiOAc & 0.4 & Trace \\
\hline 3 & 0 & $\mathrm{PCy}_{3}$ & $\mathrm{LiOAc}$ & 0.4 & 34 & 12 & 3.0 & $\mathrm{PCy}_{3}$ & $\mathrm{NaOAc}$ & 0.4 & 15 \\
\hline 4 & 3.0 & $\mathrm{PCy}_{3}$ & LiOAc & 0.0 & 26 & 13 & 3.0 & $\mathrm{PCy}_{3}$ & KOAc & 0.4 & 16 \\
\hline 5 & 3.0 & & LiOAc & 0.4 & Trace & 14 & 3.0 & $\mathrm{PCy}_{3}$ & $\mathrm{Na}_{2} \mathrm{CO}_{3}$ & 0.4 & 10 \\
\hline 6 & 3.0 & $\mathrm{PCy}_{3}$ & & 0.4 & 28 & 15 & 3.0 & $\mathrm{PCy}_{3}$ & $\mathrm{~K}_{2} \mathrm{CO}_{3}$ & 0.4 & Trace \\
\hline 7 & 1.0 & $\mathrm{PCy}_{3}$ & LiOAc & 0.4 & 45 & $16^{d}$ & 3.0 & $\mathrm{PCy}_{3}$ & LiOAc & 0.4 & 50 \\
\hline 8 & 2.0 & $\mathrm{PCy}_{3}$ & LiOAc & 0.4 & 58 & $17^{e}$ & 3.0 & $\mathrm{PCy}_{3}$ & LiOAc & 0.4 & 25 \\
\hline 9 & 3.0 & $\mathrm{PPh}_{3}$ & $\mathrm{LiOAc}$ & 0.4 & Trace & & & & & & \\
\hline
\end{tabular}

${ }^{a}$ Reaction conditons: 1a $(0.2 \mathrm{mmol}), 2 \mathrm{a}$ (10 equiv.), Pd(OAc) $)_{2}(10 \mathrm{~mol} \%)$, ligand $(25 \mathrm{~mol} \%), \mathrm{Ag}_{2} \mathrm{CO}_{3}(3.0$ equiv.), base (1.0 equiv.), TEMPO (0.4 equiv.), $101 \mathrm{kPa}$ $\mathrm{O}_{2}$, DME, $110{ }^{\circ} \mathrm{C}, 24 \mathrm{~h} . ;^{b} \mathrm{NMR}$ yield using $\mathrm{CH}_{2} \mathrm{Br}_{2}$ as internal standard (values in parentheses refers to the isolated yield); ${ }^{c}$ in absence of $\mathrm{Pd}(\mathrm{TFA}) 2 .{ }^{d}$ the reaction was conducted under air.; ${ }^{e}$ the reaction was conducted under $\mathrm{N}_{2}$ atmosphere.

Table 2 Scope of propiophenones ${ }^{a}$
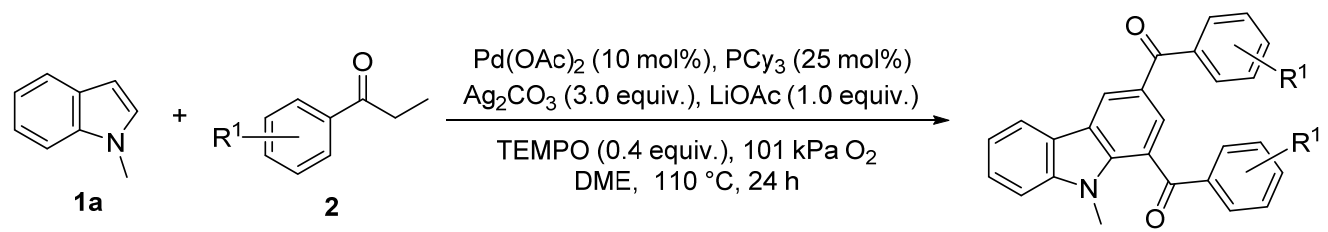

3

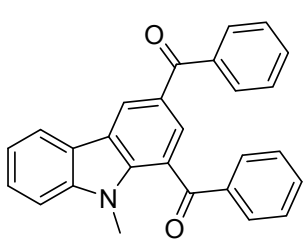

3a, $76 \%$<smiles>CCC(C)c1ccc(C(=O)c2cc(C(=O)c3ccc(Br)cc3)c3c(c2)c2ccccc2n3C)cc1</smiles>

$3 e, 52 \%$<smiles>Cn1c2ccccc2c2cc(C(=O)c3cccc(C(F)(F)F)c3)cc(C(=O)c3cccc(C(F)(F)F)c3)c21</smiles>

3i, $63 \%$<smiles>Cc1ccc(C(=O)c2cc(C(=O)c3ccc(C)cc3)c3c(c2)c2ccccc2n3C)cc1</smiles>

3b, $53 \%$<smiles>CCc1ccc(C(=O)c2cc(C(=O)c3ccc(CC)cc3)c3c(c2)c2ccccc2n3C)cc1</smiles>

$3 c, 45 \%$<smiles>CC(C)c1ccc(C(=O)c2cc(C(=O)c3ccc(C(C)C)cc3)c3c4cc(C(=O)c5ccc(C(C)C)cc5)c5ccccc5n(C)c=4c3c2)cc1</smiles>

$3 d, 53 \%$<smiles>COc1cccc(C(=O)c2cc(C(=O)c3cccc(OC)c3)c3c(c2)c2ccccc2n3C)c1</smiles>

3h, 58\%<smiles>COc1ccc(C(=O)c2cc(C(=O)c3ccc(OC)cc3)c3c(c2)c2ccccc2n3C)cc1</smiles>

3f, $57 \%^{b}$

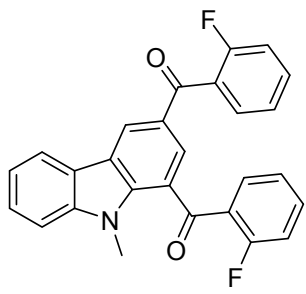

$3 \mathbf{j}, 65 \%$<smiles>Cc1cccc(C(=O)c2cc(C(=O)c3cccc(C)c3)c3c(c2)c2ccccc2n3C)c1</smiles>

3g, $61 \%$

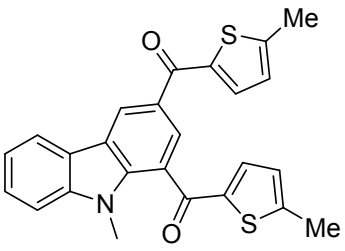

3I, $55 \%$

${ }^{a}$ Reaction conditons: 1a $\left(0.2\right.$ mmol, 1.0 equiv.), 2 (10 equiv.), $\mathrm{Pd}(\mathrm{OAc})_{2}(10 \mathrm{~mol} \%), \mathrm{PCy}_{3}(25 \mathrm{~mol} \%), \mathrm{Ag}_{2} \mathrm{CO}_{3}\left(3.0\right.$ equiv.), $\mathrm{LiOAc}_{(1.0}$ equiv.), TEMPO (0.4 equiv.), $101 \mathrm{kPa} \mathrm{O}$, DME, $110{ }^{\circ} \mathrm{C}, 24 \mathrm{~h}$. Isolated yields. ${ }^{b} 20 \mathrm{~mol} \% \mathrm{PCy}_{3}$ was used. 
Table 3 Scope of indoles
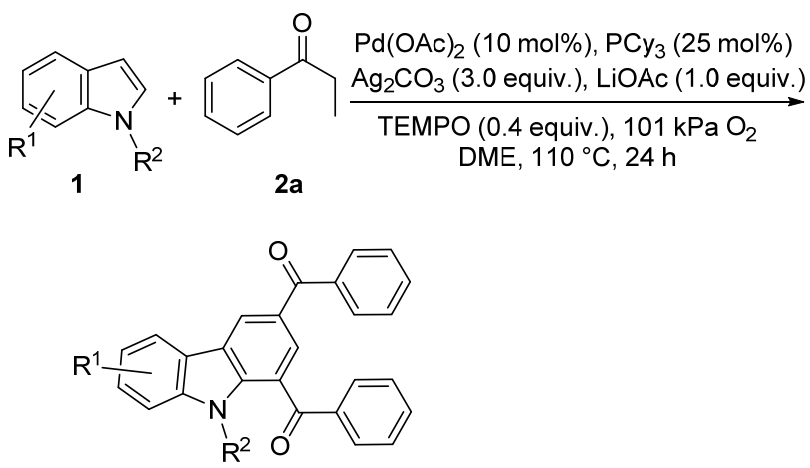

4
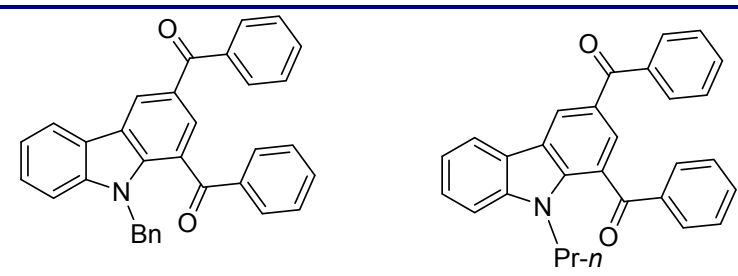

$4 a, 63 \%$<smiles>Cc1ccc2c(c1)c1cc(C(=O)c3ccccc3)cc(C(=O)c3ccccc3)c1n2C</smiles>

4c, $47 \%$<smiles>Cn1c2ccc(F)cc2c2cc(C(=O)c3ccccc3)cc(C(=O)c3ccccc3)c21</smiles>

4 e, $45 \%$

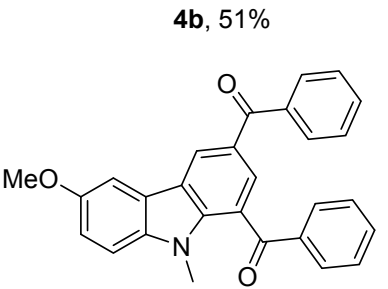

4d, $52 \%$

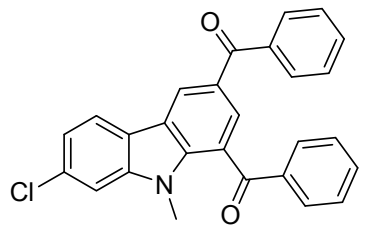

4f, $44 \%$

To probe the potential intermediates in this transformation, we examined the behavior of propiophenone (2a) in absence of indoles under standard conditions, which affored

phenyl vinyl ketone (5) in 28\% yield (Eq. 1). Moreover, the reaction of $N$-methyl indole (1a) with prepared phenyl vinyl ketone (5) provided desired product (3a) in $90 \%$ yield (Eq. $2)$. These observations provided evidence supporting that dehydrogenation of propiophenones is a key step lying in the catalytic cycle of this reaction.
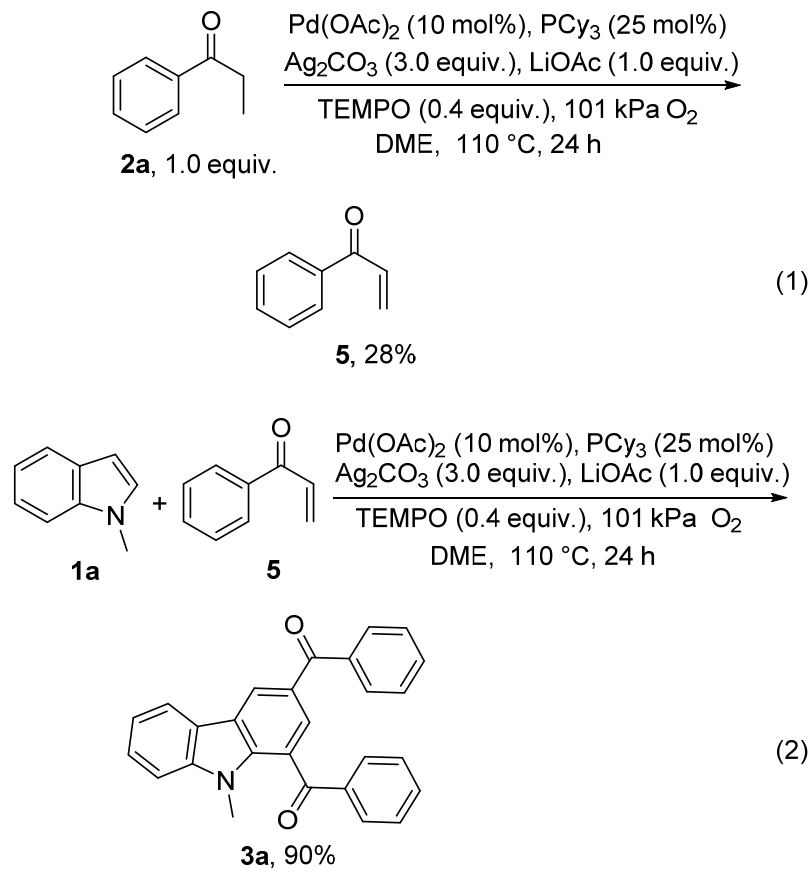

Based on above results and our related work on dehydrogenation of ketones, a proposed mechanism for this reaction is shown in Scheme 1. This reaction may involve $\mathrm{Pd}$-mediated $\mathrm{C}-\mathrm{H}$ cleavage of indoles $\mathbf{1}$ to form a palladium intermediate A. Meanwhile, $\alpha, \beta$-unsaturated ketones formed via dehydrogenation of propiophenones 2 . The intermediate $\mathbf{A}$ undergoes a Heck-type reaction with $\alpha, \beta$-unsaturated ketone to produce a $\mathrm{C}(3)$-alkenylated indole intermediate $\mathbf{C}$ and a $\operatorname{Pd}(0)$ species. The Diels-Alder reaction of the 3 -alkenylindole $\mathbf{C}$ with the second $\alpha, \beta$-unsaturated ketone, and the subsequent dehydrogenative aromatization (oxidation) of the tetrahydrocarbazole intermedi-<smiles></smiles>

Scheme 1 Proposed mechanism 
ates produce 1,3-disubstituted carbazoles 3. The oxidant $\mathrm{Ag}_{2} \mathrm{CO}_{3} / \mathrm{TEMPO} / \mathrm{O}_{2}$ facilitate the regeneration of $\mathrm{Pd}(\mathrm{II})$ catalyst.

\section{Conclusions}

In conclusion, a Pd-catalyzed direct cross-coupling reaction of indoles with ketones has been developed for the synthesis of carbazoles that are an important class of biologically active compounds. This protocol obviates the need for additional preparation steps of $\alpha, \beta$-unsaturated ketones and therefore opens up a new door to construction of carbazoles. Further studies to expand the substrate scope of this transformation to other compound are ongoing in our laboratory.

\section{Experimental section}

\subsection{Instruments and reagents}

${ }^{1} \mathrm{H}$ NMR, ${ }^{13} \mathrm{C}$ NMR and ${ }^{19} \mathrm{~F}$ NMR spectra were recorded at 400, 100.6 and $376.5 \mathrm{MHz}$, respectively. High resolution mass spectra (HRMS) were determined on a Waters Micromass GCT Premier Mass Spectrometer at Shanghai institute of organic chemistry. All Reactions were performed under nitrogen $\mathrm{N}_{2}$ with dry solvents under anhydrous conditions. DME was distilled from $\mathrm{Na}$ under a nitrogen atmosphere and stored as such. The Reagents and solvents used for experiments were purchased from Sigma-Aldrich, Acros Organics, TCI, and Alfa Aesar and used as received unless otherwise noted. 1-Benzyl- $1 H$-indole, 1-propyl-1 $H$-indole, 1,5 -dimethyl- $1 H$-indole, 5-methoxy-1methyl- $1 H$-indole, 5-fluoro-1-methyl- $1 H$-indole and 6chloro-1-methyl- $1 H$-indole were prepared according to procedures reported in the literatures. ${ }^{[17,18,19,20]}$

\subsection{Experimental method}

In a glove box, a $25 \mathrm{~mL}$ of Schlenk tube equipped with a stir bar was charged with $N$-methylindoles $(0.2 \mathrm{mmol})$, $\mathrm{Pd}(\mathrm{OAc})_{2}$ (0.002 mmol, 0.1 equiv.), $\mathrm{PCy}_{3}(0.005,0.25$ equiv.), LiOAc (0.2 mmol, 1.0 equiv.), TEMPO (0.08 mmol, 0.4 equiv.), $\mathrm{Ag}_{2} \mathrm{CO}_{3}$ (0.6 mmol, 3 equiv.). The tube was fitted with a rubber septum and removed out of the glove box. Propiophenone (2.0 mmol, 10.0 equiv.) and DME $(1 \mathrm{~mL})$ were added in turn to the Schlenk tube through the rubber septum using syringes. The tube was subjected to a vacuum and refilled with an $\mathrm{O}_{2}$ flow for three times, and then the septum was replaced with a Teflon screwcap under $\mathrm{O}_{2}$ flow (if the thiophene or the substituted propiophenone was solid, it was added to the tube in the glove box). The reaction mixture was stirred at $110{ }^{\circ} \mathrm{C}$ for $24 \mathrm{~h}$. After cooling down, the reaction mixture was diluted with $10 \mathrm{~mL}$ of ethyl ether, filtered through a pad of silica gel, followed by washing the pad of the silica gel with the same solvent $(20 \mathrm{~mL})$, concentrated under reduced pressure. The residue was then purified by flash chromatography on silica gel with $2 \% \sim 15 \%$ ethyl ether in petroleum ether as eluent to provide the corresponding products.

(9-Methyl-9H-carbazole-1,3-diyl)bis(phenylmethanone) (3a) ${ }^{[7 \mathrm{a}]}$ : White solid, $59.8 \mathrm{mg}, 76 \% .{ }^{1} \mathrm{H}$ NMR (400 MHz, $\left.\mathrm{CDCl}_{3}\right) \delta: 8.77(\mathrm{~s}, 1 \mathrm{H}), 8.15(\mathrm{~d}, J=7.7 \mathrm{~Hz}, 1 \mathrm{H}), 8.06(\mathrm{~s}$, 1H), 7.99 (d, $J=7.6 \mathrm{~Hz}, 2 \mathrm{H}), 7.85$ (d, $J=7.3 \mathrm{~Hz}, 2 \mathrm{H})$, $7.67 \sim 7.43(\mathrm{~m}, 8 \mathrm{H}), 7.34(\mathrm{t}, J=7.3 \mathrm{~Hz}, 1 \mathrm{H}), 3.68(\mathrm{~s}, 3 \mathrm{H})$; ${ }^{13} \mathrm{C}$ NMR $\left(100 \mathrm{MHz}, \mathrm{CDCl}_{3}\right) \delta: 195.68,195.66,142.50$, $140.99,138.25,137.56,133.70,133.33,131.97,130.58$, $129.83,129.69,128.67,128.60,128.23,127.97,127.31$, $127.15,125.49,124.63,122.59,122.27,120.70,120.46$, 109.49, 33.09.

(9-Methyl-9H-carbazole-1,3-diyl)bis( $p$-tolylmethanone) (3b) ${ }^{[9]}$ : White solid, $44.0 \mathrm{mg}, 53 \% .{ }^{1} \mathrm{H}$ NMR (400 MHz, $\left.\mathrm{CDCl}_{3}\right) \delta: 8.77(\mathrm{~s}, 1 \mathrm{H}), 8.17(\mathrm{~d}, J=7.5 \mathrm{~Hz}, 1 \mathrm{H}), 8.05(\mathrm{~s}$, $1 \mathrm{H}), 7.90$ (d, $J=7.5 \mathrm{~Hz}, 2 \mathrm{H}), 7.79(\mathrm{~d}, J=7.5 \mathrm{~Hz}, 2 \mathrm{H}), 7.57$ $(\mathrm{t}, J=7.2 \mathrm{~Hz}, 1 \mathrm{H}), 7.47(\mathrm{~d}, J=7.8 \mathrm{~Hz}, 1 \mathrm{H}), 7.39 \sim 7.26(\mathrm{~m}$, $5 \mathrm{H}), 3.69(\mathrm{~s}, 3 \mathrm{H}), 2.47(\mathrm{~s}, 6 \mathrm{H}) ;{ }^{13} \mathrm{C} \mathrm{NMR}(100 \mathrm{MHz}$, $\left.\mathrm{CDCl}_{3}\right) \delta: 195.54,144.78,142.70,142.47,140.80,135.54$, $135.12,130.76,130.12,129.40,129.35,128.94,127.69$, $127.05,125.19,124.50,122.64,122.47,120.57,120.45$, $109.43,32.96,21.72,21.58$.

(9-Methyl-9H-carbazole-1,3-diyl)bis((4-ethylphenyl)methanone) (3c): White solid, $40.1 \mathrm{mg}, 45 \% .{ }^{1} \mathrm{H}$ NMR (400 $\left.\mathrm{MHz}, \mathrm{CDCl}_{3}\right) \delta: 8.74(\mathrm{~d}, J=1.6 \mathrm{~Hz}, 1 \mathrm{H}), 8.16$ (d, $J=7.7$ $\mathrm{Hz}, 1 \mathrm{H}), 8.02$ (d, $J=1.5 \mathrm{~Hz}, 1 \mathrm{H}), 7.90(\mathrm{~d}, J=8.2 \mathrm{~Hz}, 2 \mathrm{H})$, $7.79(\mathrm{~d}, J=8.1 \mathrm{~Hz}, 2 \mathrm{H}), 7.58 \sim 7.54(\mathrm{~m}, 1 \mathrm{H}), 7.46(\mathrm{~d}, J=$ $8.2 \mathrm{~Hz}, 1 \mathrm{H}), 7.34 \sim 7.31(\mathrm{~m}, 5 \mathrm{H}), 3.68(\mathrm{~s}, 3 \mathrm{H}), 2.75$ (q, $J=$ $7.6 \mathrm{~Hz}, 4 \mathrm{H}), 1.32 \sim 1.27(\mathrm{~m}, 6 \mathrm{H}) ;{ }^{13} \mathrm{C} \mathrm{NMR}(100 \mathrm{MHz}$, $\left.\mathrm{CDCl}_{3}\right) \delta: 195.65,150.95,148.93,142.52,140.86,135.80$, $135.37,130.94,130.27,129.50,128.26,127.80,127.72$, $127.08,125.27,124.54,122.70,122.53,120.61,120.52$, 109.47, 33.03, 29.03, 28.93, 15.25, 15.15. HRMS (ESI) calcd for $\mathrm{C}_{31} \mathrm{H}_{28} \mathrm{NO}_{2}[\mathrm{M}+\mathrm{H}]^{+}$: 446.2115, found 446.2109.

(9-Methyl-9H-carbazole-1,3-diyl)bis((4-iso-propylphenyl)methanone) (3d): White solid, $50.1 \mathrm{mg}, 53 \% .{ }^{1} \mathrm{H}$ NMR $\left(400 \mathrm{MHz}, \mathrm{CDCl}_{3}\right) \delta: 8.79(\mathrm{~s}, 1 \mathrm{H}), 8.19(\mathrm{~d}, J=7.6 \mathrm{~Hz}, 1 \mathrm{H})$, 8.07 (s, 1H), 7.95 (d, $J=7.9 \mathrm{~Hz}, 2 \mathrm{H}), 7.84$ (d, $J=7.8 \mathrm{~Hz}$, $2 \mathrm{H}), 7.58(\mathrm{t}, J=7.5 \mathrm{~Hz}, 1 \mathrm{H}), 7.49(\mathrm{~d}, J=8.1 \mathrm{~Hz}, 1 \mathrm{H})$, $7.39 \sim 3.76(\mathrm{~m}, 5 \mathrm{H}), 3.72(\mathrm{~s}, 3 \mathrm{H}), 3.03(\mathrm{~m}, 2 \mathrm{H}), 1.33(\mathrm{~m}$, $12 \mathrm{H}) ;{ }^{13} \mathrm{C}$ NMR $\left(100 \mathrm{MHz}, \mathrm{CDCl}_{3}\right) \delta: 195.54,195.51$, $155.40,153.43,142.49,140.86,135.93,135.49,130.96$, $130.26,129.58,127.65,127.04,126.80,126.36,125.25$, $124.53,122.67,122.51,120.57,120.48,109.44,34.32$, $34.20,33.01,23.70,23.60$. HRMS (ESI) calcd for $\mathrm{C}_{33} \mathrm{H}_{32} \mathrm{NO}_{2}[\mathrm{M}+\mathrm{H}]^{+}:$474.2428, found 474.2421.

(9-Methyl-9H-carbazole-1,3-diyl)bis((4-tert-butylphenyl)methanone) (3e ${ }^{[9]}$ : White solid, $52.2 \mathrm{mg}, 52 \%$. ${ }^{1} \mathrm{H}$ NMR $\left(400 \mathrm{MHz}, \mathrm{CDCl}_{3}\right) \delta: 8.77(\mathrm{~d}, J=1.4 \mathrm{~Hz}, 1 \mathrm{H}), 8.17(\mathrm{~d}, J=$ $7.7 \mathrm{~Hz}, 1 \mathrm{H}), 8.05$ (d, $J=1.3 \mathrm{~Hz}, 1 \mathrm{H}), 7.93(\mathrm{~d}, J=8.4 \mathrm{~Hz}$, 2H), $7.82(\mathrm{~d}, J=8.3 \mathrm{~Hz}, 3 \mathrm{H}), 7.60 \sim 7.45(\mathrm{~m}, 6 \mathrm{H}), 7.34(\mathrm{t}$, $J=7.4 \mathrm{~Hz}, 1 \mathrm{H}), 3.70$ (s, 3H), $1.38(\mathrm{~d}, J=3.7 \mathrm{~Hz}, 18 \mathrm{H}) ;{ }^{13} \mathrm{C}$ NMR (100 MHz, $\left.\mathrm{CDCl}_{3}\right) \delta: 195.48,157.60,155.65,142.49$, $140.89,135.49,135.02,130.67,129.98,129.66,127.58$, $127.04,125.64,125.28,125.21,124.55,122.67,122.46$, $120.57,120.48,109.44,35.19,35.02,33.02,31.11,31.02$.

(9-Methyl-9H-carbazole-1,3-diyl)bis((4-methoxyphenyl)methanone) (3f $)^{[7 \mathrm{a}]}: \mathrm{PCy}_{3}(20 \mathrm{~mol} \%)$. White solid, 51.5 mg, 57\%. ${ }^{1} \mathrm{H}$ NMR (400 MHz, $\mathrm{CDCl}_{3}$ ) $\delta: 8.71$ (d, $J=1.5$ $\mathrm{Hz}, 1 \mathrm{H}), 8.16(\mathrm{~d}, J=7.7 \mathrm{~Hz}, 1 \mathrm{H}), 7.97 \sim 7.95(\mathrm{~m}, 2 \mathrm{H}), 7.93$ 
(s, 1H), $7.86(\mathrm{~d}, J=8.8 \mathrm{~Hz}, 2 \mathrm{H}), 7.56$ (t, $J=7.7, \mathrm{~Hz} 1 \mathrm{H})$, $7.45(\mathrm{~d}, J=8.2 \mathrm{~Hz}, 1 \mathrm{H}), 7.34(\mathrm{t}, J=7.4,1 \mathrm{H}), 6.97(\mathrm{dd}, J=$ $8.7 \mathrm{~Hz}, 6.5 \mathrm{~Hz}, 4 \mathrm{H}), 3.89$ (s, 3H), $3.89(\mathrm{~s}, 3 \mathrm{H}), 3.68(\mathrm{~s}, 3 \mathrm{H})$; ${ }^{13} \mathrm{C} \mathrm{NMR}\left(100 \mathrm{MHz}, \mathrm{CDCl}_{3}\right) \delta: 194.81,194.68,164.10$, $162.85,142.45,140.60,133.03,132.38,130.82,130.59$, $129.06,128.00,127.04,124.85,124.44,122.65,122.52$, $120.52,120.49,113.96,113.54,109.42,55.58,55.47$, 32.86 .

(9-Methyl-9H-carbazole-1,3-diyl)bis( $m$-tolylmethanone) (3g): White solid, $51.3 \mathrm{mg}, 61 \% .{ }^{1} \mathrm{H}$ NMR (400 MHz, $\left.\mathrm{CDCl}_{3}\right) \delta: 8.78(\mathrm{~d}, J=1.4 \mathrm{~Hz}, 1 \mathrm{H}), 8.16(\mathrm{~d}, J=7.7 \mathrm{~Hz}, 1 \mathrm{H})$, $8.02(\mathrm{~d}, J=1.0 \mathrm{~Hz}, 1 \mathrm{H}), 7.83(\mathrm{~s}, 1 \mathrm{H}), 7.75$ (d, $J=7.6 \mathrm{~Hz}$, $1 \mathrm{H}), 7.67(\mathrm{~s}, 1 \mathrm{H}), 7.62(\mathrm{~d}, J=6.7 \mathrm{~Hz}, 1 \mathrm{H}), 7.57(\mathrm{t}, J=7.7$ $\mathrm{Hz}, 1 \mathrm{H}), 7.46$ (dd, $J=7.7 \mathrm{~Hz}, 4.8 \mathrm{~Hz}, 2 \mathrm{H}), 7.41 \sim 7.35(\mathrm{~m}$, $4 \mathrm{H}), 3.69(\mathrm{~s}, 3 \mathrm{H}), 2.43(\mathrm{~s}, 6 \mathrm{H}) ;{ }^{13} \mathrm{C} \mathrm{NMR}(100 \mathrm{MHz}$, $\left.\mathrm{CDCl}_{3}\right) \delta: 195.96,142.50,140.99,138.61,138.33,138.14$, $137.65,134.54,132.75,130.84,130.27,129.66,128.50$, $128.14,128.00,127.44,127.14,127.12,125.40,124.66$, $122.64,122.33,120.66,120.49,109.47,33.08,21.36$, 21.31. HRMS (ESI) calcd for $\mathrm{C}_{29} \mathrm{H}_{24} \mathrm{NO}_{2}[\mathrm{M}+\mathrm{H}]^{+}$: 418.1802, found 418.1796.

(9-Methyl-9H-carbazole-1,3-diyl)bis((3-methoxyphenyl)methanone) (3h): White solid, $89.8 \mathrm{mg}, 58 \% .{ }^{1} \mathrm{H}$ NMR $\left(400 \mathrm{MHz}, \mathrm{CDCl}_{3}\right) \delta: 8.77(\mathrm{~d}, J=1.4 \mathrm{~Hz}, 1 \mathrm{H}), 8.16(\mathrm{~d}, J=$ $7.7 \mathrm{~Hz}, 1 \mathrm{H}), 8.05(\mathrm{~d}, J=1.4 \mathrm{~Hz}, 1 \mathrm{H}), 7.58(\mathrm{~s}, 1 \mathrm{H}), 7.55$ (d, $J=7.5 \mathrm{~Hz}, 1 \mathrm{H}), 7.46(\mathrm{~d}, J=8.0 \mathrm{~Hz}, 2 \mathrm{H}), 7.41 \sim 7.34(\mathrm{~m}$, $5 \mathrm{H}), 7.19(\mathrm{dd}, J=8.1 \mathrm{~Hz}, 2.0 \mathrm{~Hz}, 1 \mathrm{H}), 7.15 \sim 7.10(\mathrm{~m}, 1 \mathrm{H})$, 3.87 (s, 3H), 3.85 (s, 3H), 3.69 (s, 3H); ${ }^{13} \mathrm{C} \mathrm{NMR}(100 \mathrm{MHz}$, $\left.\mathrm{CDCl}_{3}\right) \delta: 195.52,195.47,159.88,159.53,142.50,141.02$, $139.60,138.94,129.73,129.63,129.16,127.30,127.17$, $125.50,124.64,123.92,122.63,122.53,122.24,120.73$, $120.51,120.39,118.42,114.20,114.03,109.50,55.47$, 55.40, 33.12. HRMS (ESI) calcd for $\mathrm{C}_{29} \mathrm{H}_{24} \mathrm{NO}_{4}[\mathrm{M}+\mathrm{H}]^{+}$: 450.1700 , found 450.1695 .

(9-Methyl-9H-carbazole-1,3-diyl)bis((3-(trifluoromethyl)phenyl)methanone) (3i): White solid, $67.5 \mathrm{mg}, 63 \% .{ }^{1} \mathrm{H}$ NMR (400 MHz, $\left.\mathrm{CDCl}_{3}\right) \delta: 8.78(\mathrm{~d}, J=1.4 \mathrm{~Hz}, 1 \mathrm{H}), 8.34$ $(\mathrm{s}, 1 \mathrm{H}), 8.15(\mathrm{t}, J=8.1 \mathrm{~Hz}, 2 \mathrm{H}), 8.11(\mathrm{~s}, 1 \mathrm{H}), 7.99$ (d, $J=8.6$ $\mathrm{Hz}, 2 \mathrm{H}), 7.92$ (d, $J=7.8 \mathrm{~Hz}, 1 \mathrm{H}), 7.84$ (d, $J=7.8 \mathrm{~Hz}, 1 \mathrm{H})$, $7.70 \sim 7.57(\mathrm{~m}, 3 \mathrm{H}), 7.50(\mathrm{~d}, J=8.2 \mathrm{~Hz}, 1 \mathrm{H}), 7.38(\mathrm{t}, J=7.4$ $\mathrm{Hz}, 1 \mathrm{H}), 3.72(\mathrm{~s}, 3) ;{ }^{13} \mathrm{C} \mathrm{NMR}\left(100 \mathrm{MHz}, \mathrm{CDCl}_{3}\right) \delta: 193.89$, 193.71, 142.67, 141.41, 138.87, 138.10, 133.90, 132.81, 130.08 (q, $J=3.5 \mathrm{~Hz}), 129.75,129.44,128.90,128.50$ (q, $J=3.5 \mathrm{~Hz}), 127.60,126.94$ (q, $J=3.8 \mathrm{~Hz}), 126.44$ (q, $J=$ $4.1 \mathrm{~Hz}), 125.93,125.36,122.46,121.37,121.16,120.57$, 109.71, 33.39; ${ }^{19} \mathrm{~F}$ NMR $\left(376 \mathrm{MHz}, \mathrm{CDCl}_{3}\right) \delta$ : $-62.66(\mathrm{~s}$, $3 \mathrm{~F}),-62.70(\mathrm{~s}, 3 \mathrm{~F})$. HRMS (ESI) calcd for $\mathrm{C}_{29} \mathrm{H}_{18} \mathrm{~F}_{6} \mathrm{NO}_{2}$ $[\mathrm{M}+\mathrm{H}]^{+}:$526.1234, found 526.1230.

(9-Methyl-9H-carbazole-1,3-diyl)bis((2-fluorophenyl)methanone) (3j): White solid, $67.5 \mathrm{mg}, 63 \% .{ }^{1} \mathrm{H}$ NMR (400 $\left.\mathrm{MHz}, \mathrm{CDCl}_{3}\right) \delta: 8.72(\mathrm{~d}, J=1.3 \mathrm{~Hz}, 1 \mathrm{H}), 8.17 \sim 8.10(\mathrm{~m}$, $2 \mathrm{H}), 7.84(\mathrm{td}, J=7.5 \mathrm{~Hz}, 1.7 \mathrm{~Hz}, 1 \mathrm{H}), 7.67 \sim 7.50(\mathrm{~m}, 5 \mathrm{H})$, $7.38 \sim 7.30(\mathrm{~m}, 3 \mathrm{H}), 7.24 \sim 7.14(\mathrm{~m}, 2 \mathrm{H}), 3.83(\mathrm{~s}, 3 \mathrm{H}) ;{ }^{13} \mathrm{C}$ NMR $\left(100 \mathrm{MHz}, \mathrm{CDCl}_{3}\right) \delta: 192.23,191.94,161.84(\mathrm{~d}, J=$ $255.3 \mathrm{~Hz}), 159.31$ (d, $J=255.2 \mathrm{~Hz}), 134.83$ (d, $J=8.8 \mathrm{~Hz})$, $132.73(\mathrm{~d}, J=8.2 \mathrm{~Hz}), 131.98,130.63$ (d, $J=2.9 \mathrm{~Hz})$, $129.98,127.38,127.33,127.27,127.17,126.80$ (d, $J=11.0$
Hz), 126.16, 125.21, 124.44 (d, $J=3.8 \mathrm{~Hz}), 124.23$ (d, $J=$ $3.6 \mathrm{~Hz}), 123.83,122.64,120.99,120.43,116.80$ (d, $J=21.9$ $\mathrm{Hz}), 116.17(\mathrm{~d}, J=21.8 \mathrm{~Hz}), 109.76,33.83 .{ }^{19} \mathrm{~F}$ NMR $(376$ $\left.\mathrm{MHz}, \mathrm{CDCl}_{3}\right) \delta$ : $-109.44(\mathrm{~s}, 1 \mathrm{~F}),-111.34(\mathrm{~s}, 1 \mathrm{~F})$. HRMS (ESI) calcd for $\mathrm{C}_{27} \mathrm{H}_{18} \mathrm{~F}_{2} \mathrm{NO}_{2}[\mathrm{M}+\mathrm{H}]^{+}: 426.1300$, found 426.1296.

(9-Methyl-9H-carbazole-1,3-diyl)bis((3-chlorophenyl)methanone) (3k): $\mathrm{PCy}_{3}(20 \mathrm{~mol} \%)$. White solid, $45.7 \mathrm{mg}$, $50 \% .{ }^{1} \mathrm{H}$ NMR $\left(400 \mathrm{MHz}, \mathrm{CDCl}_{3}\right) \delta: 8.76(\mathrm{~s}, 1 \mathrm{H}), 8.17(\mathrm{~d}$, $J=7.7 \mathrm{~Hz}, 1 \mathrm{H}), 7.99$ (d, $J=11.7 \mathrm{~Hz}, 2 \mathrm{H}), 7.82$ (d, $J=8.9$ $\mathrm{Hz}, 2 \mathrm{H}), 7.68$ (d, $J=7.5 \mathrm{~Hz}, 1 \mathrm{H}), 7.64 \sim 7.54(\mathrm{~m}, 3 \mathrm{H})$, $7.51 \sim 7.42(\mathrm{~m}, 3 \mathrm{H}), 7.38(\mathrm{t}, J=7.4 \mathrm{~Hz}, 3 \mathrm{H}), 3.71(\mathrm{~s}, 3 \mathrm{H})$; ${ }^{13} \mathrm{C}$ NMR $\left(100 \mathrm{MHz}, \mathrm{CDCl}_{3}\right) \delta: 194.08,193.99,142.63$, $141.26,139.89,139.14,135.16,134.62,133.69,132.04$, $130.28,130.07,129.84,129.70,129.61,128.91,127.89$, $127.50,126.68,125.81,125.13,122.52,121.55,121.05$, 120.61, 109.67, 33.36. HRMS (ESI) calcd for $\mathrm{C}_{27} \mathrm{H}_{18^{-}}$ $\mathrm{Cl}_{2} \mathrm{NO}_{2}[\mathrm{M}+\mathrm{H}]^{+}:$458.0704, found 458.0704.

(9-Methyl-9H-carbazole-1,3-diyl)bis((5-methylthiophen-2-yl)methanone) (3I): White solid, $47.2 \mathrm{mg}, 55 \% .{ }^{1} \mathrm{H}$ NMR (400 MHz, $\left.\mathrm{CDCl}_{3}\right) \delta: 8.79(\mathrm{~d}, J=1.6 \mathrm{~Hz}, 1 \mathrm{H}), 8.18$ $(\mathrm{d}, J=1.6 \mathrm{~Hz}, 1 \mathrm{H}), 8.15(\mathrm{~d}, J=7.8 \mathrm{~Hz}, 1 \mathrm{H}), 7.56 \sim 7.51(\mathrm{~m}$, 2H), 7.44 (d, $J=8.3 \mathrm{~Hz}, 1 \mathrm{H}), 7.40(\mathrm{~d}, J=3.7 \mathrm{~Hz}, 1 \mathrm{H}), 7.32$ $(\mathrm{t}, J=7.4 \mathrm{~Hz}, 1 \mathrm{H}), 6.86 \sim 6.83(\mathrm{~m}, 1 \mathrm{H}), 3.74(\mathrm{~s}, 3 \mathrm{H}), 2.59$ $(\mathrm{s}, 3 \mathrm{H}), 2.57(\mathrm{~s}, 3 \mathrm{H}) ;{ }^{13} \mathrm{C} \mathrm{NMR}\left(100 \mathrm{MHz}, \mathrm{CDCl}_{3}\right) \delta$ : $187.36,186.68,152.20,149.60,142.52,142.36,141.55$, $140.12,137.23,135.02,128.21,127.93,127.27,127.04$, $126.61,124.57,124.14,122.46,122.10,120.48,120.37$, 109.37, 32.68, 16.17, 15.91. HRMS (ESI) calcd for $\mathrm{C}_{25} \mathrm{H}_{20} \mathrm{~S}_{2} \mathrm{NO}_{2}[\mathrm{M}+\mathrm{H}]^{+}:$430.0921, found 430.0924.

(9-Benzyl-9H-carbazole-1,3-diyl)bis(phenylmethanone) (4a) ${ }^{[9]}$ : White solid, $58.2 \mathrm{mg}, 63 \% .{ }^{1} \mathrm{H}$ NMR (400 MHz, $\left.\mathrm{CDCl}_{3}\right) \delta: 8.83(\mathrm{~d}, J=1.7 \mathrm{~Hz}, 1 \mathrm{H}), 8.25(\mathrm{~d}, J=7.8 \mathrm{~Hz}, 1 \mathrm{H})$, $7.90(\mathrm{~d}, J=1.7 \mathrm{~Hz}, 1 \mathrm{H}), 7.86 \sim 7.84(\mathrm{~m}, 2 \mathrm{H}), 7.63 \sim 7.57$ $(\mathrm{m}, 3 \mathrm{H}), 7.55 \sim 7.46(\mathrm{~m}, 5 \mathrm{H}), 7.44 \sim 7.40(\mathrm{~m}, 1 \mathrm{H}), 7.34 \sim$ $7.27(\mathrm{~m}, 2 \mathrm{H}), 6.99 \sim 6.85(\mathrm{~m}, 3 \mathrm{H}), 6.68(\mathrm{~d}, J=7.2 \mathrm{~Hz}, 2 \mathrm{H})$, $5.64(\mathrm{~s}, 2 \mathrm{H}) ;{ }^{13} \mathrm{C} \mathrm{NMR}\left(100 \mathrm{MHz}, \mathrm{CDCl}_{3}\right) \delta$ : 195.64, $195.30,142.85,139.65,138.12,137.03,135.59,132.91$, $132.04,130.35,129.89,129.72,128.31,128.23,128.01$, $127.41,127.36,127.25,126.78,125.47,125.36,123.26$, $122.68,121.00,120.58,109.82,48.07$.

(9-Propyl-9H-carbazole-1,3-diyl)bis(phenylmethanone) (4b) ${ }^{[9]}$ : Yellow oil, $43.0 \mathrm{mg}, 51 \% .{ }^{1} \mathrm{H}$ NMR (400 MHz, $\left.\mathrm{CDCl}_{3}\right) \delta: 8.75(\mathrm{~d}, J=1.7 \mathrm{~Hz}, 1 \mathrm{H}), 8.16(\mathrm{~d}, J=7.8 \mathrm{~Hz}, 1 \mathrm{H})$, $7.99(\mathrm{~d}, J=1.7 \mathrm{~Hz}, 2 \mathrm{H}), 7.97 \sim 7.96(\mathrm{~m}, 1 \mathrm{H}), 7.85 \sim 7.83$ $(\mathrm{m}, 2 \mathrm{H}), 7.67 \sim 7.62(\mathrm{~m}, 1 \mathrm{H}), 7.61 \sim 7.55(\mathrm{~m}, 2 \mathrm{H}), 7.54 \sim$ $7.47(\mathrm{~m}, 5 \mathrm{H}), 7.37 \sim 7.32(\mathrm{~m}, 1 \mathrm{H}), 4.22(\mathrm{t}, J=7.6 \mathrm{~Hz}, 2 \mathrm{H})$, $1.67 \sim 1.57(\mathrm{~m}, 2 \mathrm{H}), 0.71(\mathrm{t}, J=7.4 \mathrm{~Hz}, 3 \mathrm{H}) ;{ }^{13} \mathrm{C}$ NMR $(100$ $\left.\mathrm{MHz}, \mathrm{CDCl}_{3}\right) \delta: 195.88,195.74,142.09,139.64,138.34$, $137.35,133.79,132.01,130.65,129.91,129.56,128.75$, $128.29,127.19,127.08,125.44,124.88,122.83,122.45$, 120.67, 120.51, 109.94, 46.60, 21.69, 11.08.

(6,9-Dimethyl-9H-carbazole-1,3-diyl)bis(phenylmethanone) $(4 \mathrm{c})^{[9]}$ : White solid, $38.2 \mathrm{mg}, 47 \% .{ }^{1} \mathrm{H}$ NMR (400 $\left.\mathrm{MHz}, \mathrm{CDCl}_{3}\right) \delta: 8.71(\mathrm{~s}, 1 \mathrm{H}), 8.03(\mathrm{~s}, 1 \mathrm{H}), 7.99 \sim 7.93(\mathrm{~m}$, $3 \mathrm{H}), 7.84(\mathrm{~d}, J=7.0 \mathrm{~Hz}, 2 \mathrm{H}), 7.65 \sim 7.56(\mathrm{~m}, 2 \mathrm{H}), 7.51(\mathrm{~d}$, $J=4.5 \mathrm{~Hz}, 4 \mathrm{H}), 7.37$ (q, $J=8.3 \mathrm{~Hz}, 2 \mathrm{H}), 3.66$ (s, 3H), 2.55 
(s, 3H); ${ }^{13} \mathrm{C}$ NMR $\left(100 \mathrm{MHz}, \mathrm{CDCl}_{3}\right) \delta: 195.83,195.76$, $141.22,140.90,138.42,137.69,133.70,131.95,130.65$, $130.30,129.88,129.66,128.70,128.55,128.28,127.12$, $125.58,124.52,122.79,122.24,120.44,109.25,33.19$, 21.35.

(6-Methoxy-9-methyl-9H-carbazole-1,3-diyl)bis(phenylmethanone) (4d): White solid, $43.8 \mathrm{mg}, 52 \% .{ }^{1} \mathrm{H}$ NMR (400 $\left.\mathrm{MHz}, \mathrm{CDCl}_{3}\right) \delta: 8.72(\mathrm{~d}, J=1.6 \mathrm{~Hz}, 1 \mathrm{H}), 8.02(\mathrm{~d}, J=1.6$ $\mathrm{Hz}, 1 \mathrm{H}), 7.99 \sim 7.95(\mathrm{~m}, 2 \mathrm{H}), 7.86 \sim 7.82(\mathrm{~m}, 2 \mathrm{H}), 7.66 \sim$ $7.56(\mathrm{~m}, 3 \mathrm{H}), 7.51(\mathrm{td}, J=7.5,4.1 \mathrm{~Hz}, 4 \mathrm{H}), 7.37(\mathrm{~d}, \quad J=$ $8.9 \mathrm{~Hz}, 4 \mathrm{H}), 7.19$ (dd, $J=8.9,2.5 \mathrm{~Hz}, 4 \mathrm{H}), 3.94(\mathrm{~s}, 3 \mathrm{H})$, $3.66(\mathrm{~s}, 3 \mathrm{H}) ;{ }^{13} \mathrm{C}$ NMR $\left(100 \mathrm{MHz}, \mathrm{CDCl}_{3}\right) \delta: 195.73$, $154.86,141.36,138.41,137.66,137.42,133.71,131.96$, $130.64,129.88,129.82,128.70,128.29,126.86,125.65$, $124.51,123.15,122.29,116.51,110.41,103.01,56.02$, 33.26. HRMS (ESI) calcd for $\mathrm{C}_{28} \mathrm{H}_{22} \mathrm{NO}_{3}[\mathrm{M}+\mathrm{H}]^{+}$: 420.1594, found 420.1589 .

(6-Fluoro-9-methyl-9H-carbazole-1,3-diyl)bis(phenylmethanone) (4e) $)^{[9]}$ : White solid, $36.7 \mathrm{mg}, 45 \%$. ${ }^{1} \mathrm{H}$ NMR $\left(400 \mathrm{MHz}, \mathrm{CDCl}_{3}\right) \delta: 8.68(\mathrm{~s}, 1 \mathrm{H}), 8.06(\mathrm{~s}, 1 \mathrm{H}), 7.98(\mathrm{~d}$, $J=7.5 \mathrm{~Hz}, 2 \mathrm{H}), 7.84 \sim 7.78(\mathrm{~m}, 3 \mathrm{H}), 7.67 \sim 7.58(\mathrm{~m}, 2 \mathrm{H})$, $7.51(\mathrm{q}, J=7.6 \mathrm{~Hz}, 4 \mathrm{H}), 7.40(\mathrm{dd}, J=8.8,3.9 \mathrm{~Hz}, 1 \mathrm{H})$, $7.34 \sim 7.26(\mathrm{~m}, 1 \mathrm{H}), 3.68(\mathrm{~s}, 3 \mathrm{H}) ;{ }^{13} \mathrm{C}$ NMR $(100 \mathrm{MHz}$, $\left.\mathrm{CDCl}_{3}\right) \delta: 195.56,158.14(\mathrm{~d}, J=238.4 \mathrm{~Hz}), 141.74,138.89$, $138.18,137.51,133.85,132.13,130.68,130.17,129.87$, $128.77,128.35,127.37,125.94,124.15(\mathrm{~d}, J=4.1 \mathrm{~Hz})$, $123.25(\mathrm{~d}, J=9.7 \mathrm{~Hz}), 122.65,115.07(\mathrm{~d}, J=25.6 \mathrm{~Hz})$, 110.39 (d, $J=9.0 \mathrm{~Hz}), 106.36$ (d, $J=24.2 \mathrm{~Hz}), 33.39 ;{ }^{19} \mathrm{~F}$ NMR $\left(376 \mathrm{MHz}, \mathrm{CDCl}_{3}\right) \delta$ : -122.42 .

(7-Chloro-9-methyl-9H-carbazole-1,3-diyl)bis(phenylmethanone) (4f): White solid, $37.3 \mathrm{mg}, 44 \% .{ }^{1} \mathrm{H}$ NMR (400 $\left.\mathrm{MHz}, \mathrm{CDCl}_{3}\right) \delta: 8.69(\mathrm{~d}, J=1.6 \mathrm{~Hz}, 1 \mathrm{H}), 8.04(\mathrm{t}, J=4.7 \mathrm{~Hz}$, $2 \mathrm{H}), 7.99 \sim 7.96(\mathrm{~m}, 3 \mathrm{H}), 7.82(\mathrm{dd}, J=5.2,3.3 \mathrm{~Hz}, 2 \mathrm{H})$, $7.68 \sim 7.62(\mathrm{~m}, 1 \mathrm{H}), 7.62 \sim 7.56(\mathrm{~m}, 1 \mathrm{H}), 7.56 \sim 7.47(\mathrm{~m}$, $4 \mathrm{H}), 7.46(\mathrm{~d}, J=1.6 \mathrm{~Hz}, 1 \mathrm{H}), 7.31(\mathrm{dd}, J=8.3,1.7 \mathrm{~Hz}, 1 \mathrm{H})$, $3.65(\mathrm{~s}, 3 \mathrm{H}) ;{ }^{13} \mathrm{C}$ NMR $\left(100 \mathrm{MHz}, \mathrm{CDCl}_{3}\right) \delta: 195.57$, $195.52,143.14,141.31,138.12,137.43,133.90,133.09$, $132.15,130.67,129.88,129.80,128.78,128.34,127.97$, $125.40,124.18,122.63,121.40,121.28,121.20,109.86$, 33.30. HRMS (ESI) calcd for $\mathrm{C}_{27} \mathrm{H}_{19} \mathrm{ClNO}_{2}[\mathrm{M}+\mathrm{H}]^{+}$: 424.1099, found 424.1093.

Supporting Information The copies of the ${ }^{1} \mathrm{H}$ NMR, ${ }^{19} \mathrm{~F}$ NMR, and ${ }^{13} \mathrm{C}$ NMR spectra. The Supporting Information is available free of charge via the Internet at http://siocjournal.cn/.

\section{References}

[1] (a) Zhang, F.-F.; Gan, L.-L.; Zhou, C.-H. Bioorg. Med. Chem. Lett. 2010, 20, 1881.

(b) Blunt, J. W.; Copp, B. R.; Munro, M. H. G.; Northcote, P. T.; Prinsep, M. R. Nat. Prod. Rep. 2003, $20,1$.

(c) Deslandes, S.; Chassaing, S.; Delfourne, E. Mar. Drugs 2009, 7, 754 .

(d) Maneerat, W.; Ritthiwigrom, T.; Cheenpracha, S.; Promgool, T.; Yossathera, K.; Deachathai, S.; Phakhodee, W.; Laphookhieo, S. J. Nat. Prod. 2012, 75, 741.

[2] (a) Roy, J.; Jana, A. K.; Mal, D. Tetrahedron 2012, 68, 6099. (b) Knölker, H.-J.; Reddy, K. R. Chem. Rev. 2002, 102, 4303.

(c) Schmidt, A. W.; Reddy, K. R.; Knölker, H.-J. Chem. Rev. 2012, 112,3193 .

[3] (a) Ackermann, L.; Vicente, R.; Kapdi, A. R. Angew. Chem., Int. Ed. 2009, 48, 9792.

(b) Lyons, T. W.; Sanford, M. S. Chem. Rev. 2010, 110, 1147.

(c) Liu, C.; Zhang, H.; Shi, W.; Lei, A. Chem. Rev. 2011, 111, 1780.

(d) Yeung, C. S.; Dong, V. M. Chem. Rev. 2011, 111, 1215.

[4] Zhao, J.; Larock, R. C. J. Org. Chem. 2006, 71, 5340.

[5] (a) Tsang, W. C. P.; Zheng, N.; Buchwald, S. L. J. Am. Chem. Soc. $\mathbf{2 0 0 5}, 127,14560$

(b) Jordan-Hore, J. A.; Johansson, C. C.; Beck, E. M.; Gaunt, M. J. J. Am. Chem. Soc. 2008, 130, 16184.

(c) Cho, S. H.; Yoon, J.; Chang, S. J. Am. Chem. Soc. 2011, 133, 5996.

(d) Takamatsu, K.; Hirano, K.; Satoh, T.; Miura, M. Org. Lett. 2014, 16, 2892.

[6] (a) Yamashita, M.; Horiguchi, H.; Hirano, K.; Satoh, T.; Miura, M. J. Org. Chem. 2009, 74, 7481.

(b) Jia, J.; Shi, J.; Zhou, J.; Liu, X.; Song, Y.; Xu, H. E.; Yi, W. Chem. Commun. 2015, 51, 2925.

[7] (a) Ozaki, K.; Zhang, H.; Ito, H.; Lei, A.; Itami, K. Chem. Sci. 2013, 4, 3416.

(b) Verma, A. K.; Danodia, A. K.; Saunthwal, R. K.; Patel, M.; Choudhary, D. Org. Lett. 2015, 17, 3658.

(c) Laha, J. K.; Dayal, N. Org. Lett 2015, 17, 4742.

(d) Chen, S.; Li, Y.; Ni, P.; Huang, H.; Deng, G. J. Org. Lett 2016.

[8] Grimster, N. P.; Gauntlett, C.; Godfrey, C. R.; Gaunt, M. J. Angew. Chem., Int. Ed. 2005, 44, 3125.

[9] Guo, T.; Jiang, Q.; Huang, F.; Chen, J.; Yu, Z. Org. Chem. Front. 2014, $1,707$.

[10] (a) Muzart, J. Eur. J. Org. Chem. 2010, 3779.

(b) Newhouse, T.; Turlik, A.; Chen, Y. Synlett 2016, 27, 331.

[11] (a) Nicolaou, K. C.; Gray, D. L. F.; Montagnon, T.; Harrison, S. T. Angew. Chem., Int. Ed. 2002, 41, 996.

(b) Nicolaou, K. C.; Montagnon, T.; Baran, P. S. Angew. Chem., Int. Ed. 2002, 41, 993.

(c) Nicolaou, K. C.; Montagnon, T.; Baran, P. S.; Zhong, Y. L. J. Am. Chem. Soc. 2002, 124, 2245.

(d) Uyanik, M.; Akakura, M.; Ishihara, K. J. Am. Chem. Soc. 2009, $131,251$.

(e) Nicolaou, K. C.; Zhong, Y. L.; Baran, P. S. J. Am. Chem. Soc. 2000, 122,7596 .

[12] (a) Bhattacharya, A.; DiMichele, L. M.; Dolling, U. H.; Douglas, A. W.; Grabowski, E. J. J. J. Am. Chem. Soc. 1988, 110, 3318.

(b) Walker, D.; Hiebert, J. D. Chem. Rev. 1967, 67, 153.

[13] (a) Diao, T.; Stahl, S. S. J. Am. Chem. Soc. 2011, 133, 14566.

(b) Gao, W. M.; He, Z. Q.; Qian, Y.; Zhao, J.; Huang, Y. Chem. Sci. 2012, 3, 883 .

(c) Diao, T.; Wadzinski, T. J.; Stahl, S. S. Chem. Sci. 2012, 3, 887.

(d) Diao, T.; Pun, D.; Stahl, S. S. J. Am. Chem. Soc. 2013, 135, 8205.

(e) Bigi, M. A.; White, M. C. J. Am. Chem. Soc. 2013, 135, 7831.

[14] (a) Zhang, M.; Zhang, Y.; Jie, X.; Zhao, H.; Li, G.; Su, W. Org. Chem. Front. 2014, 1, 843 .

(b) Wei, Y.; Hu, P.; Zhang, M.; Su, W. Chem. Rev. 2017.

[15] (a) Zhang, M.; Zhou, J.; Kan, J.; Wang, M.; Su, W.; Hong, M. Chem. Commun. 2010, 46, 5455.

(b) Zhou, J.; Wu, G.; Zhang, M.; Jie, X.; Su, W. Chem.-Eur. J. 2012, 18, 8032 .

(c) Zhang, M.; Hu, P.; Zhou, J.; Wu, G.; Huang, S.; Su, W. Org. Lett. 2013, 15, 1718.

(d) Shang, Y.; Jie, X.; Zhou, J.; Hu, P.; Huang, S.; Su, W. Angew. Chem. Int. Ed. 2013, 52, 1299.

[16] Jie, X.; Shang, Y.; Zhang, X.; Su, W. J. Am. Chem. Soc. 2016, 138, 5623.

[17] Xiao, B.; Li, Y. M.; Liu, Z. J.; Yang, H. Y.; Fu, Y. Chem. Commun. 2012, $48,4854$. 
[18] Klare, H. F.; Oestreich, M.; Ito, J.; Nishiyama, H.; Ohki, Y.; Tatsumi, K. J. Am. Chem. Soc. 2011, 133, 3312.

[19] Taylor, J. E.; Jones, M. D.; Williams, J. M. J.; Bull, S. D. Org. Lett. 2010, 12,5740 .
[20] Qi, T.; Qiu, W.; Liu, Y.; Zhang, H.; Gao, X.; Liu, Y.; Lu, K.; Du, C.; Yu, G.; Zhu, D. J. Org. Chem. 2008, 73, 4638.

(Li, L.; Fan, Y.) 